\title{
Clinical Relevance of Nontuberculous Mycobacteria Isolated from Sputum in a Gold Mining Workforce in South Africa: An Observational, Clinical Study
}

\author{
Clare L. van Halsema, ${ }^{1}$ Violet N. Chihota, ${ }^{2}$ \\ Nicolaas C. Gey van Pittius, ${ }^{3}$ Katherine L. Fielding, ${ }^{1}$ James J. Lewis, ${ }^{1}$ \\ Paul D. van Helden, ${ }^{3}$ Gavin J. Churchyard, ${ }^{1,2,4}$ and Alison D. Grant ${ }^{1}$ \\ ${ }^{1}$ London School of Hygiene and Tropical Medicine, Keppel Street, London WC1E 7HT, UK \\ ${ }^{2}$ The Aurum Institute, Postnet Suite No. 300, Private Bag X30500, Houghton, Johannesburg 2041, South Africa \\ ${ }^{3}$ DST/NRF Centre of Excellence for Biomedical Tuberculosis Research, MRC Centre for Molecular and Cellular Biology, \\ Division of Molecular Biology and Human Genetics, Faculty of Medicine and Health Sciences, Stellenbosch University, \\ Private Bag X1, Matieland, Cape Town 7602, South Africa \\ ${ }^{4}$ School of Public Health, University of Witwatersrand, Private Bag 3, Wits 2050, South Africa
}

Correspondence should be addressed to Clare L. van Halsema; claretaylor@doctors.org.uk

Received 23 October 2014; Revised 19 December 2014; Accepted 21 December 2014

Academic Editor: Mehdi Mirsaeidi

Copyright (C) 2015 Clare L. van Halsema et al. This is an open access article distributed under the Creative Commons Attribution License, which permits unrestricted use, distribution, and reproduction in any medium, provided the original work is properly cited.

Background. The clinical relevance of nontuberculous mycobacteria (NTM), detected by liquid more than solid culture in sputum specimens from a South African mining workforce, is uncertain. We aimed to describe the current spectrum and relevance of NTM in this population. Methods. An observational study including individuals with sputum NTM isolates, recruited at workforce tuberculosis screening and routine clinics. Symptom questionnaires were administered at the time of sputum collection and clinical records and chest radiographs reviewed retrospectively. Results. Of 232 individuals included (228 (98\%) male, median age 44 years), M. gordonae (60 individuals), M. kansasii (50), and M. avium complex (MAC: 38) were the commonest species. Of 38 MAC isolates, only $2(5.3 \%)$ were from smear-positive sputum specimens and 30/38 grew in liquid but not solid culture. MAC was especially prevalent among symptomatic, HIV-positive individuals. HIV prevalence was high: 57/74 (77\%) among those tested. No differences were found in probability of death or medical separation by NTM species. Conclusions. M. gordonae, M. kansasii, and MAC were the commonest NTM among miners with suspected tuberculosis, with most MAC from smear-negative specimens in liquid culture only. HIV testing and identification of key pathogenic NTM in this setting are essential to ensure optimal treatment.

\section{Introduction}

The nontuberculous mycobacteria (NTM) form a group of organisms diverse in many characteristics, including pathogenicity and clinical disease. They have environmental reservoirs and are associated with a broad spectrum of clinical presentations from cutaneous to lung or disseminated disease. There is uncertainty regarding clinical relevance of many of the species, particularly in settings where facility to isolate and identify them is relatively recent.
Liquid mycobacterial culture is recommended for investigation of suspected tuberculosis in resource-limited settings [1]. Compared with solid culture, time to positive culture is decreased and yield increased [2-4]. NTM accounted for $77 \%$ of the additional yield from liquid versus solid culture of smear-negative sputa in a gold mining population [2]. It is therefore important to understand the clinical relevance of NTM isolates, particularly in settings of high HIV and tuberculosis prevalence, where NTM may be isolated from large numbers of individuals in tuberculosis programmes. 


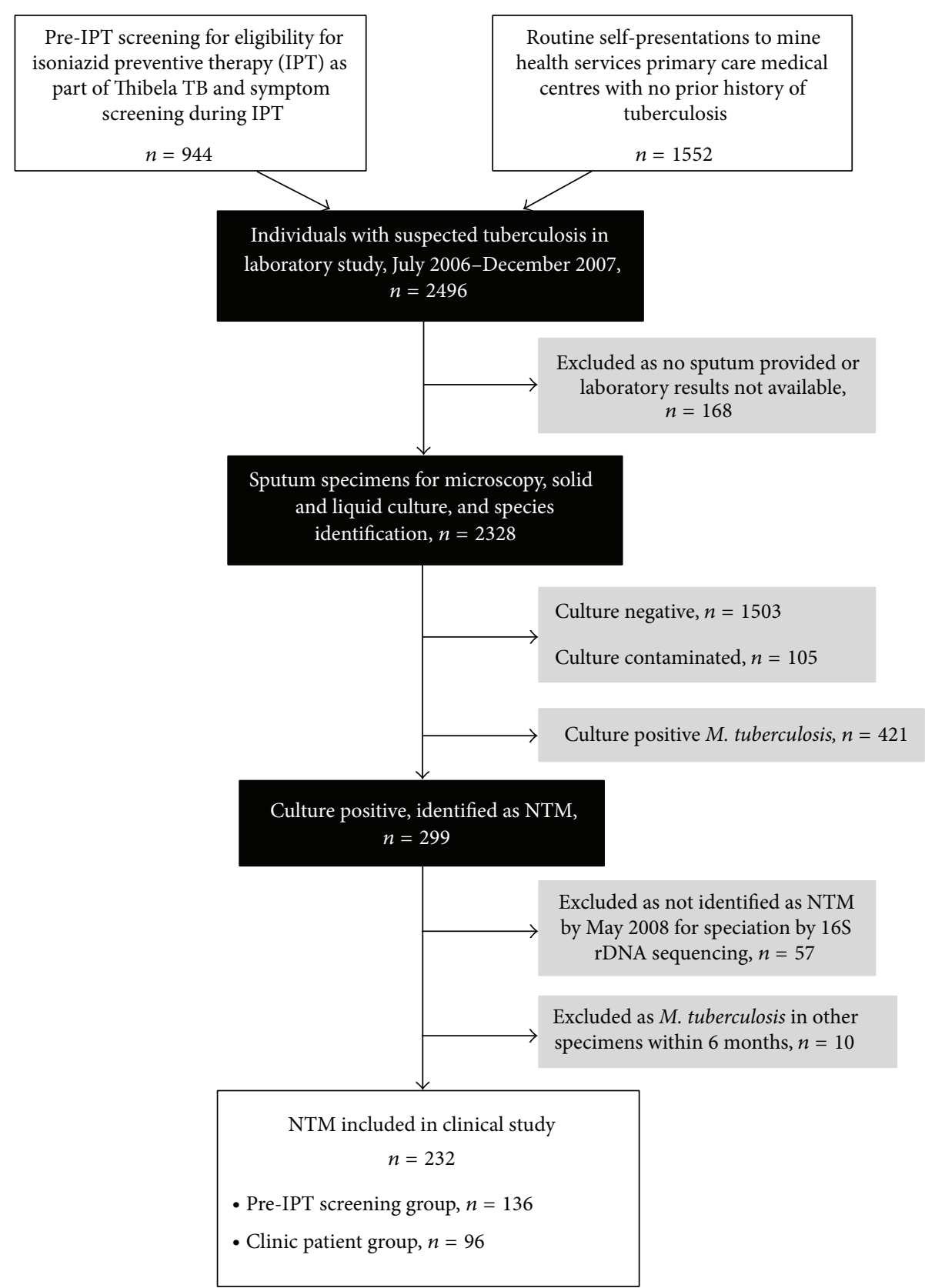

FIGURE 1: Flow chart showing sources of study participants.

To date, there is limited information from such settings, as culture and speciation facilities have not been widely available or generally applied [5].

Gold miners in South Africa have a very high incidence of tuberculosis [6] and high prevalence of HIV (estimated at $29 \%$ in 2000-2001 [7]) and silicosis [8, 9]. Previous studies in this population demonstrated disease due to NTM, particularly $M$. kansasii, M. scrofulaceum, and M. avium complex $[8,10-13]$. We have previously reported on NTM species identification in this population [14] and now describe the clinical features of those with NTM isolated from sputum, to inform laboratory and clinical practice. Previous work on molecular characteristics of M. gordonae isolates [14] led to the specific objective of reevaluating the clinical relevance of M. gordonae in this setting. We used a cross-sectional, observational study design, in the context of "Thibela TB," a cluster randomised trial of community-wide isoniazid preventive therapy (IPT) $[15,16]$.

\section{Methods}

For a laboratory study evaluating mycobacterial culture media [2], participants were recruited from two sources (Figure 1). 
(1) Screening for active tuberculosis at enrolment to Thibela TB (pre-IPT screening group): enrolment was open to the whole workforce. Screening was by symptom questionnaire and chest radiograph; nine months' IPT was given to those with negative screen. If tuberculosis was suspected at screening, sputum was taken for mycobacterial culture. All those providing sputum, regardless of tuberculosis history, were eligible for this laboratory study.

(2) Clinics run by mine health services (clinic patient group): individuals self-presented with symptoms or were referred from occupational health services, which conducted radiological screening of all employees every 6-12 months. Recruitment was restricted to individuals with no prior history of tuberculosis, aiming to strengthen diagnostic services for this group who would not routinely have been investigated with sputum culture.

Participants, recruited from July 2006 to January 2009, gave one sputum specimen, after nebulisation if necessary, and were interviewed using a standardised symptom questionnaire, which was more detailed for the clinic patient group. For this study, we included all those with NTM from sputum collected up to the end of December 2007 and with species identification using standard biochemical testing (SBT) completed by May 2008. Those with M. tuberculosis isolated within six months of the index NTM were excluded as clinical disease was likely to be due to tuberculosis. The pre-IPT screening and clinic patient groups are described separately, as characteristics of the two groups were expected to differ in terms of symptoms and stage of disease. Study participants with suspected tuberculosis were referred to the mine health service for subsequent investigation and treatment; no additional management of patients was done by investigators and this study was entirely observational, reporting routine practice in this setting.

2.1. Laboratory Methods. As described previously [2, 14], sputum smears were examined for acid fast bacilli by fluorescence microscopy; $0.5 \mathrm{~mL}$ sediment was cultured in BACTEC MGIT 960 system (BD Diagnostic Systems, Sparks, MD, USA) and a further $0.5 \mathrm{~mL}$ on Löwenstein-Jensen slants, which were incubated for up to eight weeks at $37^{\circ}$ Celsius. Mycobacteria were identified by SBT as previously described [14], anti-MPB64 assay (TAUNS, Numazu, Japan) and microscopic cording. Subsequently, NTM underwent DNA sequence amplification (using 45 amplification cycles) and analysis of the hypervariable regions of the $16 \mathrm{~S}$ rRNA gene by PCR, with referral to the RIDOM and GenBank databases for identification $[17,18]$. For specimens in which chromatograms indicated mixed NTM species, the main species is reported; numbers with mixed species are given in Table 1. A new species was defined as an isolate whose sequence did not match a known sequence in the reference databases.

2.2. Clinical Record Review. Medical and laboratory records, accessed at primary health care centres and mining hospitals, were reviewed retrospectively, using a standardised case report form in order to report routine clinical practice in this setting. Mine health services policy is to offer HIV tests to those diagnosed with tuberculosis: tests were offered to individuals in this study according to medical staff practice, with no testing done for research purposes.

2.3. Chest Radiographs. The chest radiograph closest to sputum specimen collection was read using a standardised reporting form, recording features consistent with tuberculosis, along with a silicosis score according to the International Labour Office guidelines [20]. Signs were classified as consistent with definite, probable, possible, or no disease for both active and previous tuberculosis, according to the judgment of the reader, following guidance of the chest radiograph reading and recording system [21]. The reader was masked to clinical details. For the pre-IPT group, an additional $10 \%$ of radiographs were added to further mask the reader, from those who did not have NTM isolated (no isolates or $M$. tuberculosis).

2.4. Follow-Up. Individual records were linked to mine company human resources records to establish employment and vital status of participants. Survival analysis was used to examine proportions remaining in the workforce by NTM species isolated, for majority species, from the date of sputum specimen collection. Participants were followed up from the date of sputum specimen collection until the earliest of death, medical separation (individuals leaving the workforce for medical reasons), leaving employment, or censoring date (31 December 2010).

2.5. Definitions. Individuals were considered to be HIV-positive if a positive HIV test was recorded before or up to one year following enrolment and HIV-negative if a negative HIV test (and no subsequent positive test) was recorded after or up to one year preceding enrolment. Treatment outcomes for those treated with standard tuberculosis regimens were recorded according to WHO definitions [22]. Members of the $M$. avium complex were grouped together for the most of the descriptive work presented here.

2.6. Statistical Methods. Categorical variables were compared using chi-squared or Fisher's exact test and continuous variables using the Kruskal-Wallis test. The log rank test and Kaplan-Meier curves were used to compare proportions remaining in the workforce by NTM species.

2.7. Ethical Approval. This study was approved by the Research Ethics Committees of the University of KwaZuluNatal and the London School of Hygiene and Tropical Medicine. All participants gave written or witnessed verbal consent. 
TABLE 1: Species identified using 16S sequencing, with smear status, HIV status, and CD4 counts $(n=232)$.

\begin{tabular}{|c|c|c|c|c|c|}
\hline Species & $\begin{array}{l}\text { Number isolated } \\
\text { (\% of group) }\end{array}$ & $\begin{array}{c}\text { Number }(\%) \\
\text { smear positive }\end{array}$ & $\begin{array}{c}\text { Number with mixed } \\
\text { species }^{4} \\
\end{array}$ & $\begin{array}{c}\text { HIV prevalence }{ }^{1} \\
(\%)\end{array}$ & $\begin{array}{l}\left.\text { Median } \mathrm{CD}^{2} \text { (cells/ } \mu \mathrm{L}\right) \\
\text { (range (number included)) }\end{array}$ \\
\hline \multicolumn{6}{|l|}{ Clinic patient group } \\
\hline M. avium complex & $25(26)$ & $2(8)$ & 5 & $8 / 10$ & $87(14,827[5])$ \\
\hline M. kansasii & $21(22)$ & $6(29)$ & 3 & $5 / 8$ & $85(39,763[5])$ \\
\hline M. parascrofulaceum & $9(9)$ & $1(11)$ & 0 & $3 / 3$ & $102(66,138[2])$ \\
\hline M. fortuitum & $9(9)$ & $2(22)$ & 1 & $1 / 1$ & {$[0]$} \\
\hline M. gordonae & $7(7)$ & 0 & 1 & $1 / 1$ & $195[1]$ \\
\hline New species & $7(7)$ & $1(14)$ & 0 & $3 / 3$ & $475(236,512[3])$ \\
\hline Other species & $18(19)$ & 0 & 2 & $4 / 5$ & $291.5(154,492[4])$ \\
\hline Total & 96 & $12(12.5)$ & 12 (12.5\% of 96 isolates) & $25 / 31(80.6)$ & $215.5(14,827[20])$ \\
\hline \multicolumn{6}{|l|}{ Pre-IPT $^{3}$ screening group } \\
\hline M. gordonae & $53(39)$ & $8(15)$ & 5 & $10 / 12$ & $298(66,396[8])$ \\
\hline M. kansasii & $29(21)$ & $11(38)$ & 1 & $9 / 14$ & $183.5(69,544[8])$ \\
\hline M. avium complex & $13(10)$ & 0 & 3 & $4 / 5$ & $211(92,534[4])$ \\
\hline M. parascrofulaceum & $12(9)$ & $2(17)$ & 1 & $1 / 2$ & $223[1]$ \\
\hline M. fortuitum & $11(8)$ & 0 & 1 & $3 / 5$ & $358(245,471[2])$ \\
\hline M. szulgai & $5(4)$ & 0 & 2 & $2 / 2$ & $132.5(123,142[2])$ \\
\hline New species & $3(2)$ & 0 & 0 & $0 / 0$ & {$[0]$} \\
\hline Other species & $10(7)$ & 0 & 2 & $3 / 3$ & $311(252,370[2])$ \\
\hline Total & 136 & $21(15.4)$ & 15 (11.0\% of 136 isolates) & $32 / 43(74.4)$ & $245(66,544[27])$ \\
\hline \multicolumn{6}{|c|}{$\begin{array}{l}\frac{1}{1} \text { Fisher's exact test for difference in HIV prevalence by species, both groups combined, } P=0.79 \text {. } \\
{ }^{2} \text { Median CD4 count by species for those known to be HIV-positive, combining both groups (clinic patients and IPT screening group) and including } 8 \text { species } \\
\text { compared using Kruskal-Wallis test, which showed no evidence for difference by species, } P=0.42 \text {. } \\
{ }^{3} \text { IPT: isoniazid preventive therapy. } \\
{ }^{4} \text { For specimens containing mixed NTM species on PCR sequencing, the main species is reported; minority species were not identified, but the numbers with } \\
\text { mixed species are given here. This does not apply to those with concurrent } M \text { tuberculosis, who were excluded from this studv }\end{array}$} \\
\hline
\end{tabular}

\section{Results}

3.1. Study Population. From July 2006 to December 2007, 2496 individuals were recruited and provided sputa. $720 \mathrm{spec}-$ imens $(28.8 \%)$ yielded mycobacterial growth, as described previously [2]. Of these, 421/720 (58.5\%) grew only $M$. tuberculosis and 299/720 (41.5\%) grew NTM. Fifty-seven were excluded because species identification by SBT was not completed by May 2008; 10 were excluded because of concurrent $M$. tuberculosis. Of 232 individuals included (Figure 1), 136 were enrolled through pre-IPT screening and 96 were clinic patients.

Two hundred and twenty-eight (98.3\%) of study participants were male, with median age of 44 years (interquartile range (IQR) 36, 49 years; median age 44 years [IQR 3648] for pre-IPT group and 45 years [IQR 36-50] for clinic patient group), compared with 43 years (IQR 37, 49) in the parent laboratory study [2] and 40 (IQR 31, 46) among 23299 individuals enrolling into Thibela TB at intervention clusters [23]. Median time worked in mining was 21 years (IQR 10, 28). In the pre-IPT screening group, 63/136 (46.3\%) had a history of prior tuberculosis treatment, compared with $10.7 \%$ of the 23299 enrolling at intervention clusters [23].
3.2. Mycobacterial Species and Sputum Smear Status. Species isolated are shown in Table 1 , by recruitment route. In the clinic patient group, 25 individuals, two of whom were sputum smear positive, had $M$. avium complex (12 $M$. colombiense; six M. vulneris; five $M$. intracellulare; one each of M. avium and M. chimaera). M. kansasii was isolated from 21 individuals, six of whom were sputum smear positive, and $M$. fortuitum from nine. Seven had new mycobacterial species. In the pre-IPT screening group, M. gordonae (53 individuals) was the commonest species, followed by M. kansasii (29 individuals) and then members of the M. avium complex (13 participants), principally M. colombiense (seven of 13). Eight of 53 M. gordonae, 11 of 29 M. kansasii, and none of the $M$. avium complex were isolated from smear-positive sputum specimens.

3.3. HIV Prevalence. Combining groups, HIV status was documented for 74/232 (31.9\%) individuals (Table 1). HIV prevalence among those with $M$. avium complex was $12 / 15$ (80.0\%), for M. kansasii 14/22 (63.6\%), and for M. gordonae 11/13 (84.6\%). Median CD4 count among HIV-positive individuals with $M$. avium complex was 135 cells/ $\mu \mathrm{L}$ (range 14, 827; $n=9$ ), M. kansasii 169 cells/ $\mu \mathrm{L}$ (range 39, 763; $n=13$ ), 
TABLE 2: Species distribution by HIV status, among 74 individuals with known status.

\begin{tabular}{lcc}
\hline Species & $\begin{array}{c}\text { HIV-positive } \\
\text { (total }=57) n(\%)\end{array}$ & $\begin{array}{c}\text { HIV-negative } \\
\text { (total = 17) } n(\%)\end{array}$ \\
\hline M. kansasii & $14(25)$ & $8(47)$ \\
M. avium complex & $12(21)$ & $3(18)$ \\
M. colombiense & 5 & 1 \\
M. intracellulare & 4 & 1 \\
M. vulneris & 3 & 1 \\
M. gordonae & $11(19)$ & $2(12)$ \\
M. fortuitum & $4(7)$ & $2(12)$ \\
M. parascrofulaceum & $4(7)$ & $1(6)$ \\
M. szulgai & $2(4)$ & 0 \\
New species & $3(5)$ & 0 \\
Unknown & $7(12)$ & $1(6)$ \\
\hline
\end{tabular}

and $M$. gordonae 291 cells/ $\mu \mathrm{L}$ (range 66,$396 ; n=9$ ). Species distribution by HIV status is shown in Table 2.

Of 57 HIV-positive individuals, 43 (75.4\%) were attending HIV care and $8 / 43(18.6 \%)$ had evidence of taking antiretroviral therapy.

3.4. Clinical and Radiological Features. Symptoms reported at the time of sputum specimen collection are shown in Table 3. In the clinic patient group, cough $(17 / 25 ; 68.0 \%)$ and night sweats $(13 / 25 ; 52.0 \%)$ were prevalent among those with $M$. avium complex. Cough was common for all NTM species and fever was reported most frequently by those with $M$. parascrofulaceum (6/9) and M. gordonae (4/7).

In the pre-IPT screening group, cough was less common than in the clinic patient group reported by $59 / 136(43.4 \%)$ versus $66 / 96$ (68.8\%) individuals. Cough was reported by $8 / 13$ (61.5\%) of those with M. avium complex and $26 / 53$ (49.1\%) of those with $M$. gordonae.

Combining pre-IPT screening and clinic patient groups, of 60 individuals with M. gordonae, 21 (35.0\%) reported weight loss and $14(23.3 \%)$ night sweats, while, of 38 with M. avium complex, 18 (47.4\%) reported night sweats and 14 (36.8\%) weight loss.

Chest radiographs were available for 171/232 (73.7\%) individuals. Prevalence of silicosis, grade $1 / 0$ or above, was $38 / 171$ (22.2\%) and grade $1 / 1$ or above $13 / 171$ (7.6\%). M. kansasii was most frequently associated with chest radiograph appearance suggestive of definite or probable active tuberculosis (17/34, $50.0 \%)$; corresponding figures for $M$. avium complex were $3 / 26(11.5 \%)$ and for $M$. gordonae $14 / 49$ (28.6\%). Lung cavitation was present in $47 / 171(27.5 \%)$ radiographs including $22 / 34$ (64.7\%) of those with M. kansasii, 11/49 (22.5\%) with M. gordonae, $2 / 15$ (13.3\%) with $M$. fortuitum, and 2/26 (7.7\%) with $M$. avium complex ( $\mathrm{chi}^{2} P<0.001$ for comparison of four species). Among those with cavitation, this was considered to be due to active disease in 6/11 (54.5\%) with $M$. gordonae and 8/22 (36.4\%) with M. kansasii.

M. gordonae isolates with discrepant identification on SBT were further identified by strain, as described previously,

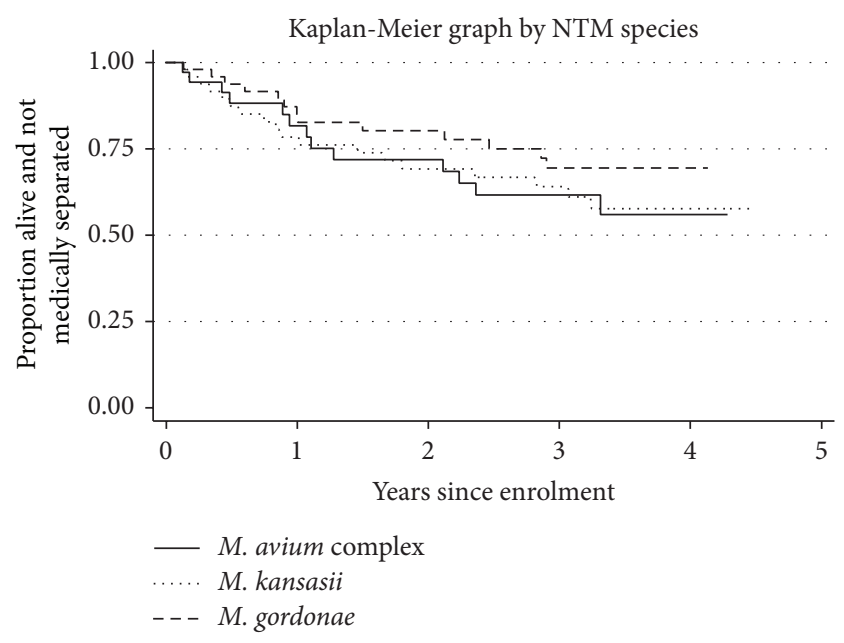

FIGURE 2: Kaplan-Meier graph showing death or medical separation by NTM species isolated for the three most prevalent species $(n=$ 138: 51 M. gordonae; 49 M. kansasii; and 38 M. avium complex).

with no differences seen between clinical features of those few isolates identified as strain D versus other M. gordonae strains [14].

3.5. Species Isolated by Culture Method. Of 38 M. avium complex isolates, $30(78.9 \%)$ were cultured on liquid but not solid culture medium. Of those 30, 9/12 individuals with known status were HIV-positive and none were sputum smear positive. For other species, 6/20 (30.0\%) M. fortuitum, 38/60 (63.3\%) M. gordonae, 12/50 (63.3\%) M. kansasii, and $15 / 21(71.4 \%)$ M. parascrofulaceum were isolated only on liquid medium (chi ${ }^{2}$ test, $P<0.001$ ). Combining all species, no significant differences in reported symptoms were observed between those cultured on solid medium and those cultured only on liquid medium (data not shown).

3.6. Outcomes and Loss to Workforce. Linking to human resources data was successful for 218/232 (94.0\%) individuals. Median follow-up time was 31.9 months (range 0.07 to 54.7 months), during which time there were 10 deaths and 63 medical separations, as shown in Table 4. At 24 months, $80.3 \%$ with $M$. gordonae, $69.2 \%$ with $M$. kansasii, and $72.9 \%$ with $M$. avium complex remained in the workforce (Table 5). Comparing the three commonest species (M. gordonae; $M$. kansasii; $M$. avium complex), log rank test for differences in proportions remaining in the workforce gave $P=0.47$ (Figure 2).

\section{Discussion}

In this mining population with high HIV prevalence, NTM were common in sputum culture of those with suspected tuberculosis. M. gordonae, M. kansasii, and M. avium complex were the commonest species, with $M$. avium complex 
TABLE 3: Symptoms reported by route of recruitment to study and species of nontuberculous mycobacterium isolated $(n=232)$.

\begin{tabular}{|c|c|c|c|c|c|c|c|c|}
\hline Species & $\begin{array}{l}\text { Number } \\
\text { isolated }\end{array}$ & $\begin{array}{l}\text { Number } \\
\text { reporting } \\
\text { cough } \\
n(\%)\end{array}$ & $\begin{array}{l}\text { Number } \\
\text { reporting } \\
\text { night } \\
\text { sweats }{ }^{1} \\
n(\%)\end{array}$ & $\begin{array}{c}\text { Number } \\
\text { reporting } \\
\text { haemoptysis } \\
n(\%)\end{array}$ & $\begin{array}{c}\text { Number } \\
\text { reporting } \\
\text { weight } \\
\text { loss } \\
n(\%)\end{array}$ & $\begin{array}{l}\text { Number } \\
\text { reporting } \\
\text { fever } \\
n(\%)\end{array}$ & $\begin{array}{l}\text { Number } \\
\text { reporting any } \\
\text { symptom } \\
n(\%)\end{array}$ & $\begin{array}{l}\text { Number with chest } \\
\text { radiograph classified } \\
\text { as definite or possible } \\
\text { active tuberculosis } \\
n / \text { number with chest } \\
\text { radiograph available }\end{array}$ \\
\hline \multicolumn{9}{|l|}{ Clinic patient group } \\
\hline M. avium complex & 25 & $17(68)$ & $13(52)$ & $4(16)$ & $11(44)$ & $11(44)$ & $19(76)$ & $2 / 14$ \\
\hline M. kansasii & 21 & $11(52)$ & $9(43)$ & $1(5)$ & $10(48)$ & $9(43)$ & $14(67)$ & $4 / 9$ \\
\hline M. parascrofulaceum & 9 & $8(89)$ & $7(78)$ & 0 & $8(89)$ & $6(67)$ & $9(100)$ & $1 / 7$ \\
\hline M. fortuitum & 9 & $7(78)$ & $5(56)$ & 0 & $5(56)$ & $3(33)$ & $7(78)$ & $1 / 5$ \\
\hline M. gordonae & 7 & $5(71)$ & $2(29)$ & 0 & $4(57)$ & $4(57)$ & $6(86)$ & $1 / 3$ \\
\hline New species & 7 & $5(71)$ & $3(43)$ & $1(14)$ & $2(29)$ & $4(57)$ & $5(71)$ & $0 / 5$ \\
\hline Other species & 18 & $13(72)$ & $11(61)$ & $3(17)$ & $7(39)$ & $10(56)$ & $15(83)$ & $1 / 7$ \\
\hline Total & 96 & $66(69)$ & $50(52.1)$ & $9(9.4)$ & $47(49.0)$ & $47(49.0)$ & $75(78.1)$ & $10 / 50$ \\
\hline \multicolumn{9}{|l|}{ Pre-IPT ${ }^{3}$ screening group } \\
\hline M. gordonae & 53 & $26(49)$ & $12(23)$ & $\S$ & $17(32)$ & $\S$ & $34(64)$ & $13 / 46$ \\
\hline M. kansasii & 29 & $8(28)$ & $2(7.0)$ & $\S$ & $6(21)$ & $\S$ & $12(41)$ & $13 / 25$ \\
\hline M. avium complex & 13 & $8(62)$ & $5(39)$ & $\S$ & $3(23)$ & $\S$ & $9(69)$ & $1 / 12$ \\
\hline M. parascrofulaceum & 12 & $2(17)$ & $2(17)$ & $\S$ & $4(33)$ & $\S$ & $6(50)$ & $4 / 11$ \\
\hline M. fortuitum & 11 & $5(46)$ & $3(27)$ & $\S$ & $1(9)$ & $\S$ & $5(46)$ & $5 / 10$ \\
\hline M. szulgai & 5 & $4(80)$ & $1(20)$ & $\S$ & $2(40)$ & $\S$ & $4(80)$ & $2 / 5$ \\
\hline New species & 3 & $1(33)$ & $1(33)$ & $\S$ & $1(33)$ & $\S$ & $1(33)$ & $1 / 2$ \\
\hline Other species & 10 & $5(50)$ & $1(10)$ & $\$$ & $5(50)$ & $\$$ & $6(60)$ & $1 / 10$ \\
\hline Total & 136 & $59(43.4)$ & 27 (19.9) & $\S$ & $39(28.7)$ & $\$$ & $77(56.6)$ & $40 / 121$ \\
\hline
\end{tabular}

${ }^{1}$ Night sweats were defined by the wording of the question put to study participants: "Do you have drenching night sweats? (Sweat so much at night that clothes/pillows are soaking wet?)."

${ }^{2}$ Weight loss was defined by the wording of the question put to study participants: "Do you have unintentional weight loss? (In the last 6 months have your clothes become looser?)."

${ }^{3}$ IPT: isoniazid preventive therapy.

${ }^{\S}$ Data not available as questionnaire used in pre-IPT screening for tuberculosis did not include this question.

TABLE 4: Deaths and medical separations, from recruitment until end of 2010, median 32 months $(n=218)$.

\begin{tabular}{lccc}
\hline NTM species & $\begin{array}{c}\text { Death } \\
n \text { (row \%) }\end{array}$ & $\begin{array}{c}\text { Medical } \\
\text { separation } \\
n \text { (row \%) }\end{array}$ & $\begin{array}{c}\text { Total } \\
\text { number of } \\
\text { individuals }\end{array}$ \\
\hline M. gordonae & 0 & $13(26)$ & 51 \\
M. kansasii & $2(4)$ & $16(33)$ & 49 \\
M. avium complex & $1(3)$ & $12(32)$ & 38 \\
M. parascrofulaceum & $2(11)$ & $2(11)$ & 19 \\
M. fortuitum & $1(5)$ & $11(58)$ & 19 \\
M. szulgai & 0 & $1(20)$ & 5 \\
New species & $1(10)$ & $1(10)$ & 10 \\
Other species & $3(11)$ & $7(26)$ & 27 \\
\hline Total & $10(4.6)$ & $63(28.9)$ & 218 \\
\hline
\end{tabular}

particularly prevalent among symptomatic individuals presenting to routine health services and $M$. gordonae most common in the pre-IPT screening group.
TABLE 5: Probability of remaining in the workforce during follow-up for individuals with the three predominant NTM species.

\begin{tabular}{lccc}
\hline Species & $\begin{array}{c}6 \text { months } \\
(\%, 95 \% \mathrm{CI})\end{array}$ & $\begin{array}{c}12 \text { months } \\
(\%, 95 \% \mathrm{CI})\end{array}$ & $\begin{array}{c}24 \text { months } \\
(\%, 95 \% \mathrm{CI})\end{array}$ \\
\hline $\begin{array}{l}\text { M. gordonae } \\
\text { M. kansasii }\end{array}$ & $87.8(82.0-98.0)$ & $85.0(91.0-92.5)$ & $80.3(65.5-89.3)$ \\
$\begin{array}{l}\text { M. avium } \\
\text { complex }\end{array}$ & $88.3(71.7-95.4)$ & $81.7(63.7-91.4)$ & $72.9(52.9-94.4)$ \\
\hline
\end{tabular}

HIV prevalence was $77.0 \%$ where known. Importantly, though this study population comprised individuals with suspected tuberculosis, a minority had HIV status documented, suggesting that testing coverage is poor and that those tested may not be representative of all those with NTM. It is possible that those tested have higher prevalence of HIV than those not tested, as there may have been clinical reasons for offering tests. However, routine testing in this setting is essential to improve care and it is very likely that diagnoses of HIV are being missed. Silicosis grade $1 / 1$ or above (definite silicosis) 
was found in $7.6 \%$ of individuals, compared with $2.6 \%$ in the parent study Thibela TB [16], which would be expected in a subpopulation with NTM, as silicosis is a known risk factor [8].

Symptoms varied by recruitment method, as expected, with cough more prevalent among those self-presenting to routine health services than those undergoing screening. Lung cavitation was more prevalent among those with $M$. kansasii than those with $M$. avium complex, which would be expected, as $M$. avium complex more typically causes disseminated disease in the context of advanced HIV-related immunosuppression and sputum isolates may represent disseminated, rather than exclusively pulmonary, disease. It is not possible to definitively diagnose disseminated disease without further sampling, but, with low CD 4 counts, systemic symptoms, and chest radiographs without cavitation, it is likely that some of this group had disseminated M. avium complex, with the organism isolated from respiratory specimens here. Similarly, radiographs were reported as consistent with active tuberculosis less frequently in those with $M$. avium complex. There was a higher than expected proportion with cavitation on chest radiograph among those with M. gordonae. This is difficult to interpret with limited clinical data available and cavitation may have been a result of previous or other diseases. We did not detect differences in proportions remaining in the workforce between groups of individuals with the three commonest NTM species, although there is a suggestion of lower retention in the workforce among those with $M$. avium complex and $M$. kansasii. The study design did not include a comparator group without NTM and from these data alone we can only conclude that loss to the workforce for health reasons among those with NTM isolated from sputum is substantial. However, from the mining population participating in Thibela TB, mortality was $0.91 / 100$ person-years and combined mortality and medical separation were 4.34/100 person-years [16], suggesting higher combined mortality and medical separation among those with NTM than the workforce overall, which may be due to mycobacterial disease or untreated HIV. Differences by species may have been detected with larger numbers.

The distribution of NTM species differs from that found in previous studies in this population. In the 1990s, $M$. kansasii and M. scrofulaceum were reported to be the commonest species $[8,11]$, whereas this study found higher proportions of $M$. avium complex and M. gordonae, with notable absence of $M$. scrofulaceum using $16 S$ sequencing. This is likely to be largely due to newer speciation methods: organisms identified by SBT as M. scrofulaceum in this study were almost all subsequently identified as M. gordonae by $16 \mathrm{~S}$ sequencing [14]. The higher proportion of M. avium complex may be due to the use of liquid culture, as suggested by laboratory studies in this population [2] and elsewhere [3,4], and may also be due to increased HIV prevalence in this population: from $1.4 \%$ among those with sexually transmitted infections in 1991 [24] to $19.0 \%$ among those with NTM in 1993-6 [11,25] and 29\% in a representative sample of the workforce in 2000-1 [7]. The median age of the population does not appear to have increased, estimated at 41 years in
2001 [7] and 40 years among those enrolling at Thibela TB intervention clusters [23], although the median age among those with NTM was higher.

M. gordonae was more prevalent in the pre-IPT screening group than in clinic patients, perhaps because, in the context of active case finding, not all isolates will be genuine pathogens. M. gordonae is well documented to be a laboratory and tap-water contaminant [19]. However, in this group of individuals with suspected tuberculosis, $M$. gordonae was associated with systemic symptoms (night sweats, weight loss) in some, with radiological abnormalities in 14/51 (27.5\%), and with HIV infection in $11 / 15$ (73.3\%). It has previously been reported to cause disseminated disease in advanced HIV [26-28] and may be pathogenic in some individuals here, with isolates from sputum indicating pulmonary or more widespread disease. Laboratory and clinical records would be expected to have detected concurrent M. tuberculosis or bacterial infections if present. Longer term, close follow-up of these individuals was not incorporated into the study design. Without more clinical data and follow-up, firm conclusions on M. gordonae in this population should not be drawn, but those with M. gordonae and clinical symptoms or abnormal radiology warrant further investigation for other pathologies, particularly HIV infection, and repeat culture of sputum and other relevant samples.

M. avium complex was particularly common among symptomatic individuals in the clinic patient group, who had high prevalence of HIV infection. M. avium complex is known to be common where it is sought and appropriate diagnostics used in HIV-positive populations and disease is associated with low CD4 cell counts [29-32]. These individuals in particular require further investigation and specific treatment, with early antiretroviral therapy and cotrimoxazole prophylaxis. It is noteworthy that $30 / 38 \mathrm{M}$. avium complex isolates grew in liquid but not solid culture media and none of these 30 were smear positive, suggesting that $M$. avium complex infection may be underdiagnosed in settings of high HIV prevalence where liquid culture medium is not used. Pulmonary isolates may reflect disseminated disease and liquid culture will be needed to diagnose this infection in settings where the Xpert MTB/RIF assay is used as first line diagnostic test for tuberculosis. There was a notable absence of $M$. avium, with other $M$. avium complex subspecies found (M. colombiense, M. vulneris, and $M$. intracellulare). This may reflect immunosuppression, as M. colombiense is closely related to M. avium and has been isolated from HIV-positive individuals previously [33] or may reflect geographical variation in NTM species [34].

Mining populations have long been known to be at risk of NTM infection and disease [12] and to some extent results presented here are relevant primarily to this population. However, comparisons of species distribution, HIV prevalence, and clinical features are relevant to other settings in which HIV and tuberculosis are prevalent and liquid mycobacterial culture media are used. Similar results have been seen in Southeast Asia in a study showing high prevalence of NTM isolated by liquid culture of sputum among HIV-positive individuals, with M. kansasii most frequent in 
TABLE 6: American Thoracic Society criteria for lung disease due to NTM (adapted from [19]).

Criteria for diagnosis of lung disease due to NTM

(1) Pulmonary symptoms, nodular or cavitary opacities on chest radiograph, or an HRCT scan that shows multifocal bronchiectasis with multiple small nodules

And

(2) Appropriate exclusion of other diagnoses

(1) Positive culture results from at least two separate expectorated sputum samples

Or

Microbiological

(2) Positive culture results from at least one bronchial wash or lavage

Or

(3) Transbronchial o other lung biopsy with mycobacterial histopathological features (granulomatous

inflammation or AFB) and positive culture for NTM or biopsy showing mycobacterial histopathological features

and one or more sputum or bronchial washings that are culture positive for NTM

those with pulmonary disease and M. avium complex among those with disseminated disease [35].

The clinic patient group was restricted to individuals with no prior tuberculosis treatment, in order to maximise the impact of the parent laboratory study on routine health services. In addition, the clinic patient group was more symptomatic than the pre-IPT screening group and there are likely to have been differences in disease spectrum between these groups. For this reason, characteristics of the two groups are presented separately. In addition, despite excluding individuals with $M$. tuberculosis isolated from other specimens within six months of the NTM, we cannot definitively exclude culture negative tuberculosis.

ATS criteria for lung disease due to NTM [19] include, amongst other criteria shown in Table 6, a requirement for NTM to be isolated from expectorated sputum samples on more than one occasion. We were not able to apply these criteria in this study because of its retrospective design. This is a limitation of this study. However, authors of the ATS criteria, primarily intended to guide diagnosis of lung disease, are clear that they were written with the United States setting in mind and are not validated in other settings. Among miners in South Africa, these criteria have been shown to be difficult to apply even in prospective studies, because of the requirement for repeated sampling and detailed imaging and frequent use of presumptive antimycobacterial treatment [10] in a programme designed for tuberculosis detection and treatment. Such repeat intensive laboratory diagnostics are not part of the national guidelines for tuberculosis control in South Africa [36].

\section{Conclusions}

In conclusion, M. avium complex, largely found among symptomatic, smear-negative individuals, will be underdiagnosed where liquid culture is not used. Newer culture techniques are advisable wherever feasible, although there are of course cost and infrastructure obstacles to widespread use. Key pathogenic organisms should be identified where possible, so that specific treatment can be given if required. HIV testing coverage was poor and prevalence among those with known HIV status was high. Improved HIV testing strategies are required for those being investigated for tuberculosis in this and other settings of high HIV prevalence.

\section{Conflict of Interests}

The authors declare that there is no conflict of interests regarding the publication of this paper.

\section{Authors' Contribution}

Clare L. van Halsema contributed to study design and implementation, data analysis, and paper writing; Violet N. Chihota contributed to study design, parent study implementation, laboratory work, and paper writing; Nicolaas C. Gey van Pittius carried out laboratory work, contributed microbiology advice, and reviewed the paper; Katherine L. Fielding contributed to study design, parent study design and implementation, epidemiological input, and paper review; James J. Lewis contributed to data management, parent study implementation, statistical and epidemiological advice, and paper review; Paul D. van Helden contributed to laboratory and study design advice and reviewed the paper; Gavin J. Churchyard contributed to study design of this and parent studies, epidemiological advice, and paper review; Alison D. Grant contributed to study concept and design, epidemiological advice, parent study design and implementation, and paper writing.

\section{Acknowledgments}

Thibela TB was funded by the Consortium to Respond Effectively to the AIDS and TB Epidemics (CREATE), with grants from the Bill and Melinda Gates Foundation, and the Safety in Mines Research Advisory Committee (South Africa). Violet N. Chihota was supported by NIH Fogarty ICORTA TB/AIDS (Grants 5U2RTW007370 and 5U2RTW007373). Clare L. van Halsema was funded by a grant from the Colt Foundation, UK. Alison D. Grant was supported by a Public Health Career Scientist Award from the Department of Health, UK. Katherine L. Fielding is part-funded by the 
Biostatistics Core of CREATE. James J. Lewis was fully funded by the Biostatistics Core of CREATE. The authors thank the staff of mine health services and The Aurum Institute for assistance in finding clinical records.

\section{References}

[1] World Health Organization, "The use of liquid medium for culture and drug susceptibility testing (DST) in low- and medium-income settings," Summary Report of the Expert Group Meeting on the Use of Liquid Culture Systems, World Health Organization, Geneva, Switzerland, 2007.

[2] V. N. Chihota, A. D. Grant, K. Fielding et al., "Liquid vs. solid culture for tuberculosis: performance and cost in a resourceconstrained setting," The International Journal of Tuberculosis and Lung Disease, vol. 14, no. 8, pp. 1024-1031, 2010.

[3] M. Muyoyeta, J. A. Schaap, P. de Haas et al., "Comparison of four culture systems for Mycobacterium tuberculosis in the Zambian national reference laboratory," International Journal of Tuberculosis and Lung Disease, vol. 13, no. 4, pp. 460-465, 2009.

[4] M. L. Katila, P. Katila, and R. Erkinjuntti-Pekkanen, "Accelerated detection and identification of mycobacteria with MGIT 960 and COBAS AMPLICOR systems," Journal of Clinical Microbiology, vol. 38, no. 3, pp. 960-964, 2000.

[5] K. Gopinath and S. Singh, "Non-Tuberculous mycobacteria in TB-endemic countries: are we neglecting the danger?" PLoS Neglected Tropical Diseases, vol. 4, no. 4, article e615, 2010.

[6] C. L. van Halsema, K. L. Fielding, V. N. Chihota, J. J. Lewis, G. J. Churchyard, and A. D. Grant, "Trends in drug-resistant tuberculosis in a gold-mining workforce in South Africa, 2002-2008," International Journal of Tuberculosis and Lung Disease, vol. 16, no. 7, pp. 967-973, 2012.

[7] J. J. Lewis, S. Charalambous, J. H. Day et al., "HIV infection does not affect active case finding of tuberculosis in South African gold miners," American Journal of Respiratory and Critical Care Medicine, vol. 180, no. 12, pp. 1271-1278, 2009.

[8] E. L. Corbett, G. J. Churchyard, T. Clayton et al., "Risk factors for pulmonary mycobacterial disease in south african gold miners: a case-control study," The American Journal of Respiratory and Critical Care Medicine, vol. 159, no. 1, pp. 94-99, 1999.

[9] E. L. Corbett, G. J. Churchyard, T. C. Clayton et al., "HIV infection and silicosis: the impact of two potent risk factors on the incidence of mycobacterial disease in South African miners," AIDS, vol. 14, no. 17, pp. 2759-2768, 2000.

[10] E. L. Corbett, L. Blumberg, G. J. Churchyard et al., "Nontuberculous mycobacteria: defining disease in a prospective cohort of South African miners," American Journal of Respiratory and Critical Care Medicine, vol. 160, no. 1, pp. 15-21, 1999.

[11] E. L. Corbett, M. Hay, G. J. Churchyard et al., "Mycobacterium kansasii and M. scrofulaceum isolates from HIV-negative South African gold miners: incidence, clinical significance and radiology," International Journal of Tuberculosis and Lung Disease, vol. 3, no. 6, pp. 501-507, 1999.

[12] R. L. Cowie, "The mycobacteriology of pulmonary tuberculosis in South African gold miners," Tubercle, vol. 71, no. 1, pp. 39-42, 1990.

[13] P. Sonnenberg, J. Murray, J. R. Glynn, R. G. Thomas, P. GodfreyFaussett, and S. Shearer, "Risk factors for pulmonary disease due to culture-positive M. tuberculosis or nontuberculous mycobacteria in South African gold miners," European Respiratory Journal, vol. 15, no. 2, pp. 291-296, 2000.
[14] V. N. Chihota, C. L. van Halsema, A. D. Grant et al., "Spectrum of non-tuberculous mycobacteria identified using standard biochemical testing vs. $16 \mathrm{~S}$ sequencing," The International Journal of Tuberculosis and Lung Disease, vol. 17, no. 2, pp. 267-269, 2013.

[15] K. L. Fielding, A. D. Grant, R. J. Hayes, R. E. Chaisson, E. L. Corbett, and G. J. Churchyard, “Thibela TB: design and methods of a cluster randomised trial of the effect of communitywide isoniazid preventive therapy on tuberculosis amongst gold miners in South Africa," Contemporary Clinical Trials, vol. 32, no. 3, pp. 382-392, 2011.

[16] G. J. Churchyard, K. L. Fielding, J. J. Lewis et al., "A trial of mass isoniazid preventive therapy for tuberculosis control," The New England Journal of Medicine, vol. 370, no. 4, pp. 301-310, 2014.

[17] D. A. Benson, I. Karsch-Mizrachi, D. J. Lipman, J. Ostell, and E. W. Sayers, "GenBank," Nucleic Acids Research, vol. 39, no. 1, pp. D32-D37, 2011.

[18] D. Harmsen, S. Dostal, A. Roth et al., "RIDOM: comprehensive and public sequence database for identification of Mycobacterium species," BMC Infectious Diseases, vol. 3, article 26, 2003.

[19] D. E. Griffith, T. Aksamit, B. A. Brown-Elliott et al., "An official ATS/IDSA statement: diagnosis, treatment, and prevention of nontuberculous mycobacterial diseases," American Journal of Respiratory and Critical Care Medicine, vol. 175, no. 4, pp. 367416, 2007.

[20] International Labour Office, Guidelines for the Use of the ILO Classification of Radiographs for Pneumoconiose, Occupational Safety and Health Series, International Labour Office, Geneva, Switzerland, 2000.

[21] S. den Boon, E. D. Bateman, D. A. Enarson et al., "Development and evaluation of a new chest radiograph reading and recording system for epidemiological surveys of tuberculosis and lung disease," International Journal of Tuberculosis and Lung Disease, vol. 9, no. 10, pp. 1088-1096, 2005.

[22] World Health Organization, Treatment of Tuberculosis: Guidelines for National Programmes, World Health Organization, Geneva, Switzerland, 3rd edition, 2003.

[23] G. J. Churchyard, K. L. Fielding, J. J. Lewis, V. N. Chihota, Y. Hanifa, and A. D. Grant, "Symptom and chest radiographic screening for infectious tuberculosis prior to starting isoniazid preventive therapy: yield and proportion missed at screening," AIDS, vol. 24, no. 5, pp. S19-S27, 2010.

[24] E. L. Corbett, S. Charalambous, K. Fielding et al., "Stable incidence rates of tuberculosis (TB) among human immunodeficiency virus (HIV)-negative South African gold miners during a decade of epidemic HIV-associated TB," The Journal of Infectious Diseases, vol. 188, no. 8, pp. 1156-1163, 2003.

[25] E. L. Corbett, G. J. Churchyard, M. Hay et al., "The impact of HIV infection on Mycobacterium kansasii disease in South African gold miners," American Journal of Respiratory and Critical Care Medicine, vol. 160, no. 1, pp. 10-14, 1999.

[26] J. Chan, J. C. McKitrick, and R. S. Klein, "Mycobacterium gordonae in the acquired immunodeficiency syndrome," Annals of Internal Medicine, vol. 101, no. 3, p. 400, 1984.

[27] K. D. Lessnau, S. Milanese, and W. Talavera, "Mycobacterium gordonae: a treatable disease in HIV-positive patients," Chest, vol. 104, no. 6, pp. 1779-1785, 1993.

[28] E. Bonnet, P. Massip, R. Bauriaud, L. Alric, and J.-C. Auvergnat, "Disseminated Mycobacterium gordonae infection in a patient infected with human immunodeficiency virus," Clinical Infectious Diseases, vol. 23, no. 3, pp. 644-645, 1996.

[29] C. F. Gilks, R. J. Brindle, C. Mwachari et al., "Disseminated Mycobacterium avium infection among HIV-infected patients 
in Kenya," Journal of Acquired Immune Deficiency Syndromes and Human Retrovirology, vol. 8, no. 2, pp. 195-198, 1995.

[30] P. C. Karakousis, R. D. Moore, and R. E. Chaisson, "Mycobacterium avium complex in patients with HIV infection in the era of highly active antiretroviral therapy," The Lancet Infectious Diseases, vol. 4, no. 9, pp. 557-565, 2004.

[31] C. A. Pettipher, A. S. Karstaedt, and M. Hopley, "Prevalence and clinical manifestations of disseminated Mycobacterium avium complex infection in South Africans with acquired immunodeficiency syndrome," Clinical Infectious Diseases, vol. 33, no. 12, pp. 2068-2071, 2001.

[32] S. D. Nightingale, L. T. Byrd, P. M. Southern, J. D. Jockusch, S. X. Cal, and B. A. Wynne, "Incidence of Mycobacterium aviumintracellulare complex bacteremia in human immunodeficiency virus-positive patients," Journal of Infectious Diseases, vol. 165, no. 6, pp. 1082-1085, 1992.

[33] M. I. Murcia, E. Tortoli, M. C. Menendez, E. Palenque, and M. J. Garcia, "Mycobacterium colombiense sp. nov., a novel member of the Mycobacterium avium complex and description of MAC$\mathrm{X}$ as a new ITS genetic variant," International Journal of Systematic and Evolutionary Microbiology, vol. 56, no. 9, pp. 2049-2054, 2006.

[34] W. Hoefsloot, J. Van Ingen, C. Andrejak et al., "The geographic diversity of nontuberculous mycobacteria isolated from pulmonary samples: an NTM-NET collaborative study," European Respiratory Journal, vol. 42, no. 6, pp. 1604-1613, 2013.

[35] K. D. McCarthy, K. P. Cain, K. L. Winthrop et al., "Nontuberculous mycobacterial disease in patients with HIV in Southeast Asia," American Journal of Respiratory and Critical Care Medicine, vol. 185, no. 9, pp. 981-988, 2012.

[36] Department of Health, Republic of South Africa. National Tuberculosis Management Guidelines, 2008, http://www.who .int/hiv/pub/guidelines/south_africa_tb.pdf. 


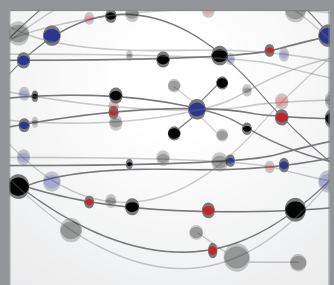

The Scientific World Journal
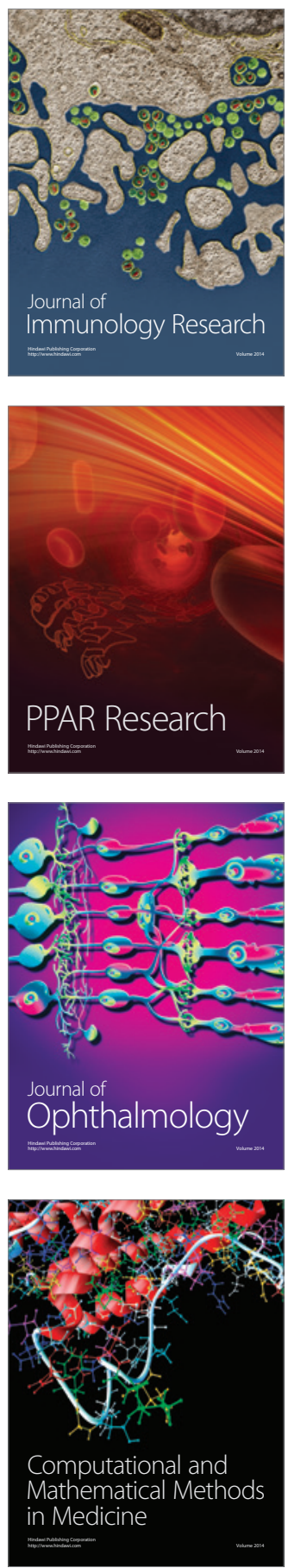

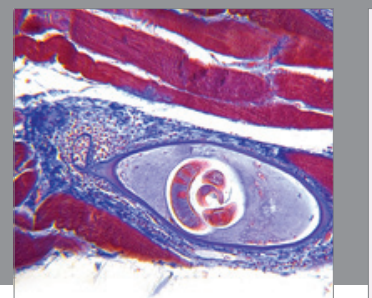

Gastroenterology

Research and Practice
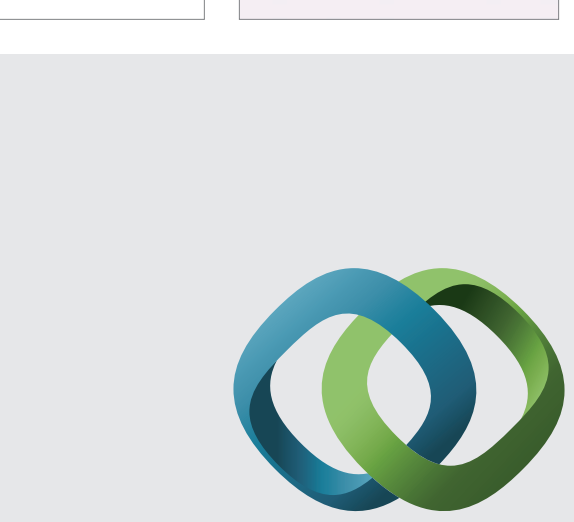

\section{Hindawi}

Submit your manuscripts at

http://www.hindawi.com
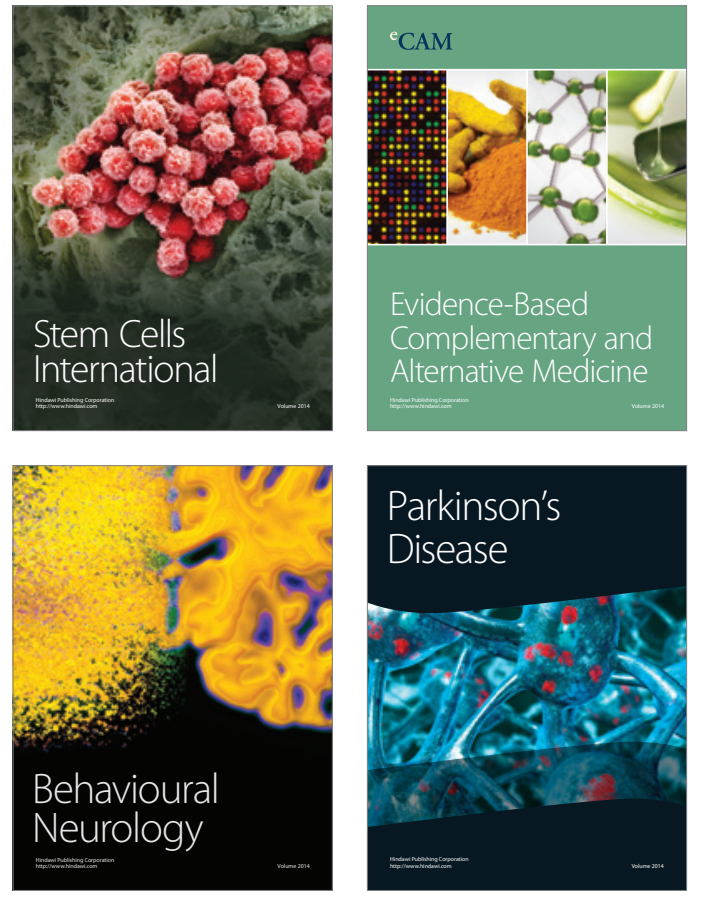
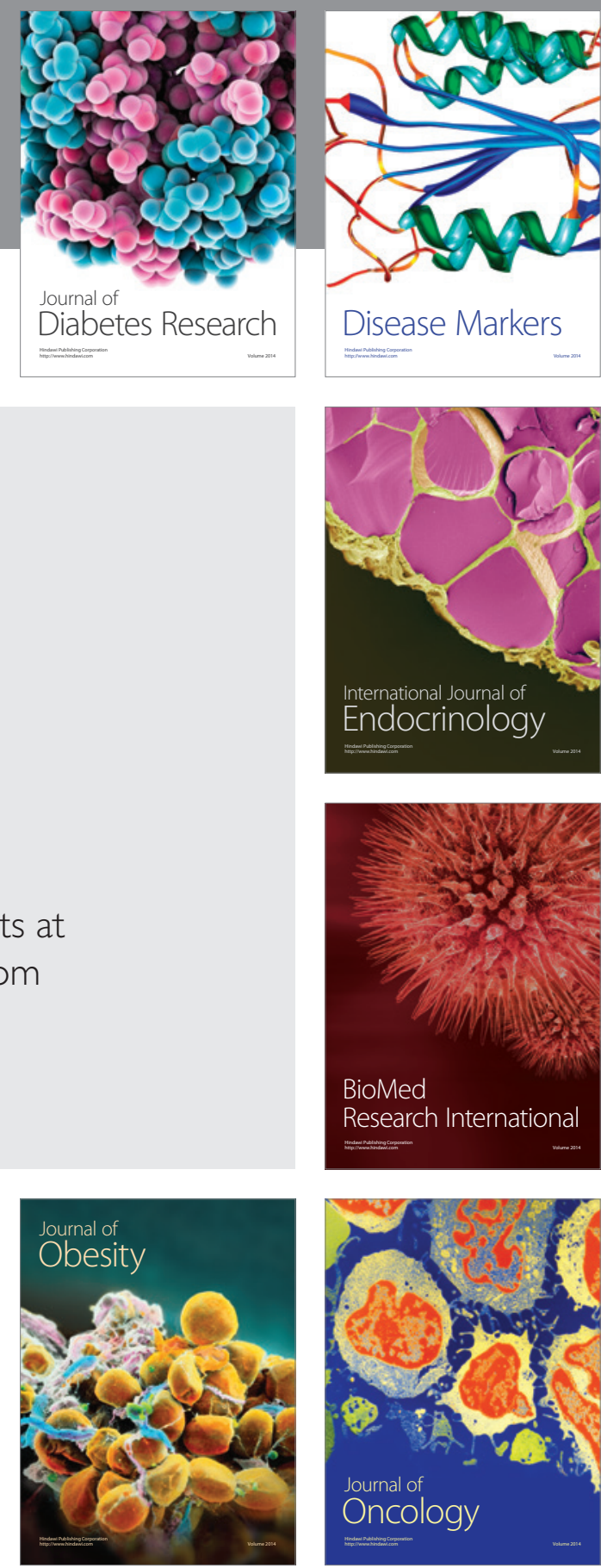

Disease Markers
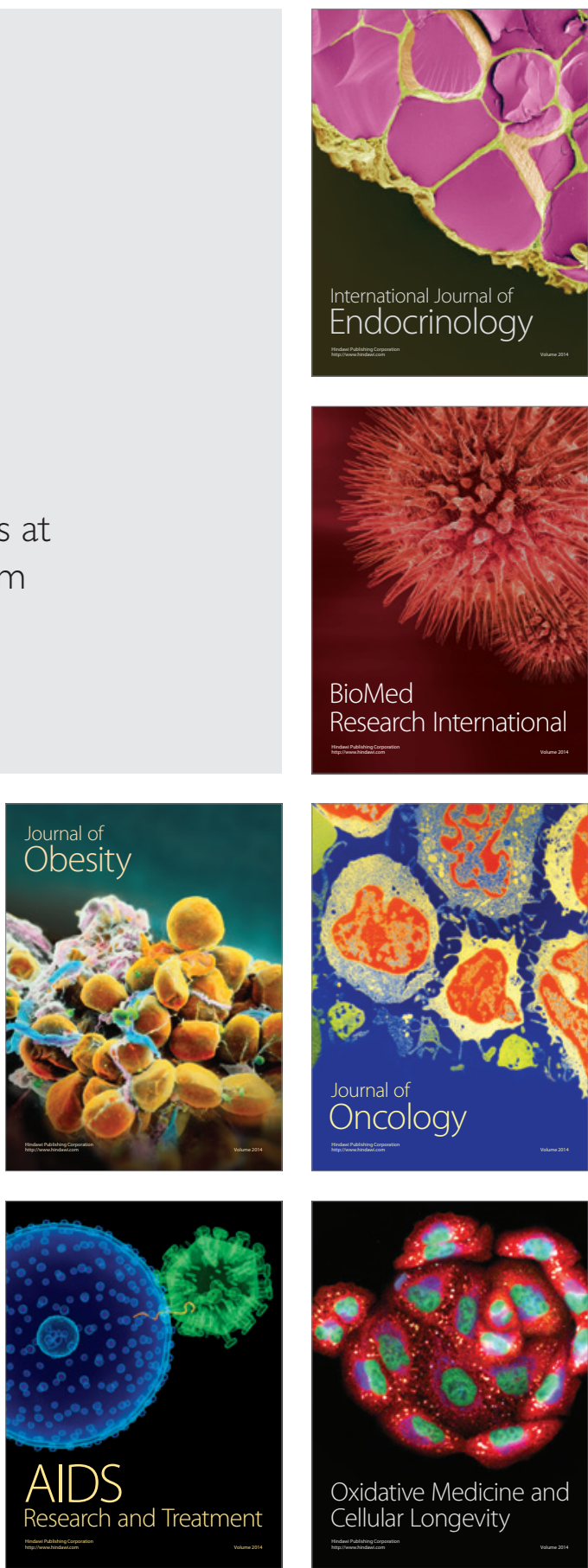